\title{
Producción, nutrientes, eutrofización y cianobacterias en Uruguay: armando el rompecabezas
}

Production, nutrients, eutrophication and cyanobacteria blooms in Uruguay: putting puzzle pieces together

Produção, nutrientes, eutrofização e cianobactérias no Uruguai: montando o quebra-cabeça

Guillermo Goyenola ${ }^{1,}{ }^{*}$ https://orcid.org/0000-0001-7688-5341

Carla Kruk $^{2,3}$ https://orcid.org/0000-0003-0760-1186

Néstor Mazzeo ${ }^{1,4}$ https://orcid.org/0000-0002-3090-2617

Alejandro Nario ${ }^{5}$ https://orcid.org/0000-0002-7081-4662

Carlos Perdomo ${ }^{6}$ https://orcid.org/0000-0002-9770-4260

Claudia Piccini ${ }^{7}$ https://orcid.org/0000-0002-2762-1953

Mariana Meerhoff ${ }^{1}$ https://orcid.org/0000-0001-6482-1332

*Autor de contacto: goyenola@gmail.com

${ }^{1}$ Departamento de Ecología y Gestión Ambiental, Centro Universitario Regional del Este, UDELAR, Maldonado, Uruguay

${ }^{2}$ IECA, Facultad de Ciencias, UDELAR, Montevideo, Uruguay

${ }^{3}$ EFSA, Centro Universitario Regional del Este, UDELAR, Rocha, Uruguay

${ }^{4}$ Instituto SARAS, Maldonado, Uruguay

${ }^{5}$ Asociación Ivy, Montevideo, Uruguay

${ }^{6}$ Departamento de Suelos y Aguas, Facultad de Agronomía, UDELAR, Montevideo, Uruguay

${ }^{7}$ Departamento de Microbiología, Instituto de Investigaciones Biológicas Clemente Estable, Montevideo, Uruguay

Recepción: 11 Agosto 2020 Aprobación: 04 Abril 2021

Esta obra está bajo una Licencia Creative Commons Atribución-NoComercial 4.0 Internacional. 
Resumen: Si bien las causas y consecuencias de la eutrofización son múltiples, este artículo analiza aspectos fundamentales en el contexto actual en Uruguay, con especial énfasis en las floraciones cianobacterianas como una de las consecuencias más frecuentes y notorias. El agravamiento de la eutrofización y sus síntomas, que se predicen con el cambio climático; las claves para la reducción de las pérdidas difusas de fósforo hacia el agua superficial en suelos bajo uso agropecuario; y los cambios actuales y futuros en la gobernanza del agua y la gestión ambiental, son algunos de los temas más importantes considerados. En el análisis se identifican también múltiples desafíos, tanto en la generación de conocimiento como en la gestión ambiental asociada.

Palabras clave: contaminación, fósforo, agricultura, intensificación sostenible, gobernanza.

Abstract: Despite there are multiple causes and consequences of eutrophication, this article analyses key aspects in the current context of Uruguay, emphasizing on cyanobacterial blooms as one of the most frequent and noticeable consequences. The worsening of eutrophication and its symptoms predicted with climate change, the keys for the reduction of diffuse losses of phosphorus with surface runoff on agricultural lands and the ongoing and future changes of water governance and management of the problem are some of the most important issues considered here. In this analysis, we also identify several challenges, both to fill knowledge gaps and in the arena of environmental management.

Keywords: pollution, phosphorus, agriculture, sustainable intensification, governance.

Resumo: Embora as causas e consequências da eutrofização sejam múltiplas, este artigo analisa aspectos fundamentais no contexto atual no Uruguai, com ênfase especial nas florações de cianobactérias como uma das consequências mais frequentes e notórias. O agravamento da eutrofização e seus sintomas previstos pelas mudanças climáticas, as chaves para reduzir as perdas difusas de fósforo para as águas superficiais em solos sob uso agrícola e as mudanças atuais e futuras na governança da água e gestão ambiental, são algumas das questões mais importantes consideradas. A análise também identifica múltiplos desafios, tanto na geração de conhecimento como na gestão ambiental associada.

Palavras-chave: contaminação, fósforo, agricultura, intensificação sustentável, governança.

\section{INTRODUCCIÓN}

La eutrofización es, junto con la fragmentación de los cursos de agua, la problemática más seria y extendida de los ecosistemas acuáticos a nivel mundial y regional (O'Farrell, et al., 2019; Quirós, et al., 2005; Smith, et al., 2006). La misma es favorecida por la ineficiencia en el uso y el manejo de nutrientes en diversas actividades de origen antrópico (Haygarth, et al., 2005). En Uruguay, la 
intensificación de la eutrofización fue diagnosticada en las últimas décadas del siglo pasado (De León y Yunes, 2001; Mazzeo, et al., 2002; Pintos, et al., 1986; Sommaruga, et al., 1995). Sin embargo, esta problemática no fue percibida por la población hasta las crisis del agua potable en el área metropolitana asociada a la ciudad de Montevideo en 2013 y en Maldonado en 2015, y la afectación generalizada de las playas en 2019 (González-Madina, et al., 2018; Kruk, et al., 2019; Olano, et al., 2019). Hoy la eutrofización es un fenómeno generalizado que afecta tanto a ecosistemas de agua dulce (Aubriot, et al., 2017; Bonilla, et al., 2015; Goyenola, et al., 2017), como al estuario del Río de la Plata y la costa oceánica (Kruk, et al., 2019; Nagy, et al., 2002).

Si bien las causas y consecuencias de la eutrofización son múltiples, este artículo se enfoca en los aspectos más relevantes en Uruguay: la producción agropecuaria como el mayor contribuyente de nutrientes, las floraciones cianobacterianas como una de las principales respuestas y sus posibles efectos a través de la producción de toxinas, las claves para la reducción de las pérdidas difusas de fósforo, los procesos por los cuales se espera un agravamiento ante el cambio climático, y las fortalezas y debilidades de los ámbitos de gestión de la problemática. El objetivo de este artículo es contribuir a una reflexión interdisciplinaria constructiva para una mejor gestión ambiental a escala país. Esta reflexión es relevante también para la región, dado que el sureste de América del Sur ha experimentado el mismo proceso de intensificación productiva sobre el bioma de pampas y campos desde el año 2000 (Modernel, et al., 2016).

\section{FORZANTES DE LA CONTAMINACIÓN POR NUTRIENTES}

\section{Actividad agropecuaria e intensificación del uso del suelo}

La producción de alimento es la actividad antrópica que más nutrientes aporta a los ecosistemas acuáticos a nivel mundial (Moss, 2008). El impacto ocurre como una consecuencia no deseada del mal uso de insumos agrícolas y de suplementos alimenticios para ganado y animales de granja, así como también de cambios en la movilización y las vías de transporte de nutrientes (Cordell, et al., 2009; Fowler, et al., 2013; Quinton, et al., 2010; Sims, et al., 1998). Asimismo, en general los sistemas productivos eliminan o debilitan los filtros naturales provistos por los humedales y ecosistemas riparios (Stutter, et al., 2012).

En Uruguay, sólo para el período 2000-2017, se estimó una intensificación agrícola-ganadera de un 65\% de la superficie terrestre (Gazzano, et al., 2019). Sin embargo, con mayor o menor intensidad, prácticamente todo el territorio puede considerarse bajo actividad productiva agrícola-ganadera (CLAES, et al., 2008), por lo que los niveles basales de nutrientes (previos a los impactos antrópicos) ya no pueden registrarse. 
El análisis de testigos de sedimento de cuerpos de agua de Uruguay correspondientes al Holoceno (últimos 10.000 años) demuestra la aceleración reciente del proceso de eutrofización y su vínculo con actividades antrópicas (García-Rodríguez, et al., 2002; Inda, et al., 2016). Por otra parte, en una comparación de grupos de pequeños arroyos de cabecera bajo usos del suelo contrastantes (agricultura, lechería y ganadería extensiva), los menores niveles de nutrientes se registraron en las cuencas bajo uso ganadero extensivo $(\leq 1$ cabeza. ha-1) (Tabla 1). En dicho estudio la fracción disuelta de fósforo predominó sobre la fracción particulada (Goyenola, et al., 2015), lo que dificulta asociar su movilización exclusivamente al proceso de erosión del suelo. Esto concuerda con otros estudios nacionales (Barreto, et al., 2014; Lizarralde, et al., 2016; Lizarralde, et al., 2014; Perdomo, et al., 2015).

Tabla 1. Concentraciones de fósforo y nitrógeno total (PT y NT; $\mu \mathrm{g} \cdot \mathrm{L}^{-1}$ ) en el agua de arroyos en cuencas con diferente uso del suelo predominante, indicando valores mínimos, medianos y máximos.

\begin{tabular}{|c|c|c|c|c|c|c|c|}
\hline $\begin{array}{l}\text { Intensidad de } \\
\text { uso del suelo }\end{array}$ & $\begin{array}{l}\text { Uso del suelo } \\
\text { principal }\end{array}$ & $\begin{array}{c}\text { PT } \\
\text { mínima }\end{array}$ & $\begin{array}{c}\text { PT } \\
\text { mediana }\end{array}$ & $\begin{array}{l}\text { PT } \\
\text { máxima }\end{array}$ & $\begin{array}{l}\text { NT } \\
\text { mínima }\end{array}$ & $\begin{array}{c}\text { NT } \\
\text { mediana }\end{array}$ & $\begin{array}{l}\text { NT } \\
\text { máxima }\end{array}$ \\
\hline Baja & $\begin{array}{l}\text { Ganadero extensivo } \\
\qquad(\mathrm{n}=10 ; i+\mathrm{v})\end{array}$ & 12 & 67 & 530 & 263 & 636 & 3393 \\
\hline Alta & $\begin{array}{l}\text { Agrícola intensivo } \\
\qquad(\mathrm{n}=12 ; \mathrm{i}+\mathrm{v})\end{array}$ & 70 & 157 & 640 & 365 & 1061 & 4563 \\
\hline Alta & $\begin{array}{c}\text { Agrícola-lechero } \\
(\mathrm{n}=9 ; \mathrm{i}+\mathrm{v})(1 \text { tambo) }\end{array}$ & 60 & 110 & 425 & 406 & 1267 & 3497 \\
\hline Muy alta & $\begin{array}{c}\text { Agrícola-lechero } \\
\text { ( } \mathrm{n}=1 ; 24 \text { muestreos } \\
\text { quincenales) (4 tambos) }\end{array}$ & 271 & 1210 & 4436 & 240 & 1614 & 5155 \\
\hline
\end{tabular}

Fuentes: Goyenola, 2016; Goyenola, et al., 2015; Goyenola, et al., 2020. "n" corresponde al número de microcuencas/arroyos estudiados. i+v: muestreos en invierno 2011 y verano 2012.

El hecho de que los aportes de las actividades agrícola-ganaderas sean de origen difuso condiciona la ocurrencia de la eutrofización en todo el territorio. A estos aportes se suman las entradas provenientes de fuentes puntuales, como la actividad industrial y la urbanización, 0 incluso de sitios con grandes aglomeraciones animales (tambos y feedlots). Esto genera concentraciones elevadas de nutrientes en el agua, espacialmente más localizadas.

En este sentido, la mayor parte de los ecosistemas acuáticos estudiados excede largamente la concentración de $25 \mu \mathrm{g} \mathrm{P} \mathrm{L}-1$ de fósforo total (PT) prevista como 
valor límite superior en la normativa vigente (Decreto 253/79 y modificativos, Uruguay, 1979). Los muestreos realizados por la ex Dirección Nacional de Medio Ambiente (DINAMA), actual Dirección Nacional de Control y Evaluación Ambiental (DINACEA), en ríos de mayor orden revelan valores de PT muy altos (Figura 1). Lo mismo ocurre con los lagos someros (tipo de lago natural presente en Uruguay) y los embalses (construidos represando arroyos y ríos) usados como fuente de agua para potabilizar. Por ejemplo, en la Laguna del Sauce (Maldonado) se han registrado concentraciones de PT en el entorno de 80-100 $\mu \mathrm{g} . \mathrm{L}^{-1}$ (Steffen e Inda, 2010), mientras que en Laguna del Cisne (Canelones) la menor concentración medida desde el año 2000 a la fecha fue de $275 \mu \mathrm{g} . \mathrm{L}^{-1}$ (Goyenola, et al., 2017).

El límite de $25 \mu \mathrm{g}$ PT L-1, establecido en la normativa, puede considerarse exigente para las características locales. Con variaciones según las características climáticas, la geología y la estructura de las tramas tróficas acuáticas en cada sistema, en general los síntomas de la eutrofización son evidentes a partir del entorno de 50-60 $\mathrm{\mu g}$. PT L L-1, mientras que a partir de concentraciones cercanas a $100 \mu \mathrm{g} \cdot \mathrm{L}^{-1}$ los síntomas pueden expresarse en toda su magnitud (Dolman, et al., 2012). Esto es particularmente notorio en cuerpos de aguas quietas, ya que el impacto es mayor cuando el tiempo de residencia del agua es mayor. Al mismo tiempo, el riesgo de eutrofización y la magnitud de los síntomas es mayor en lagos someros que en lagos profundos (Carlson, 1977; Dodds, 2006). 


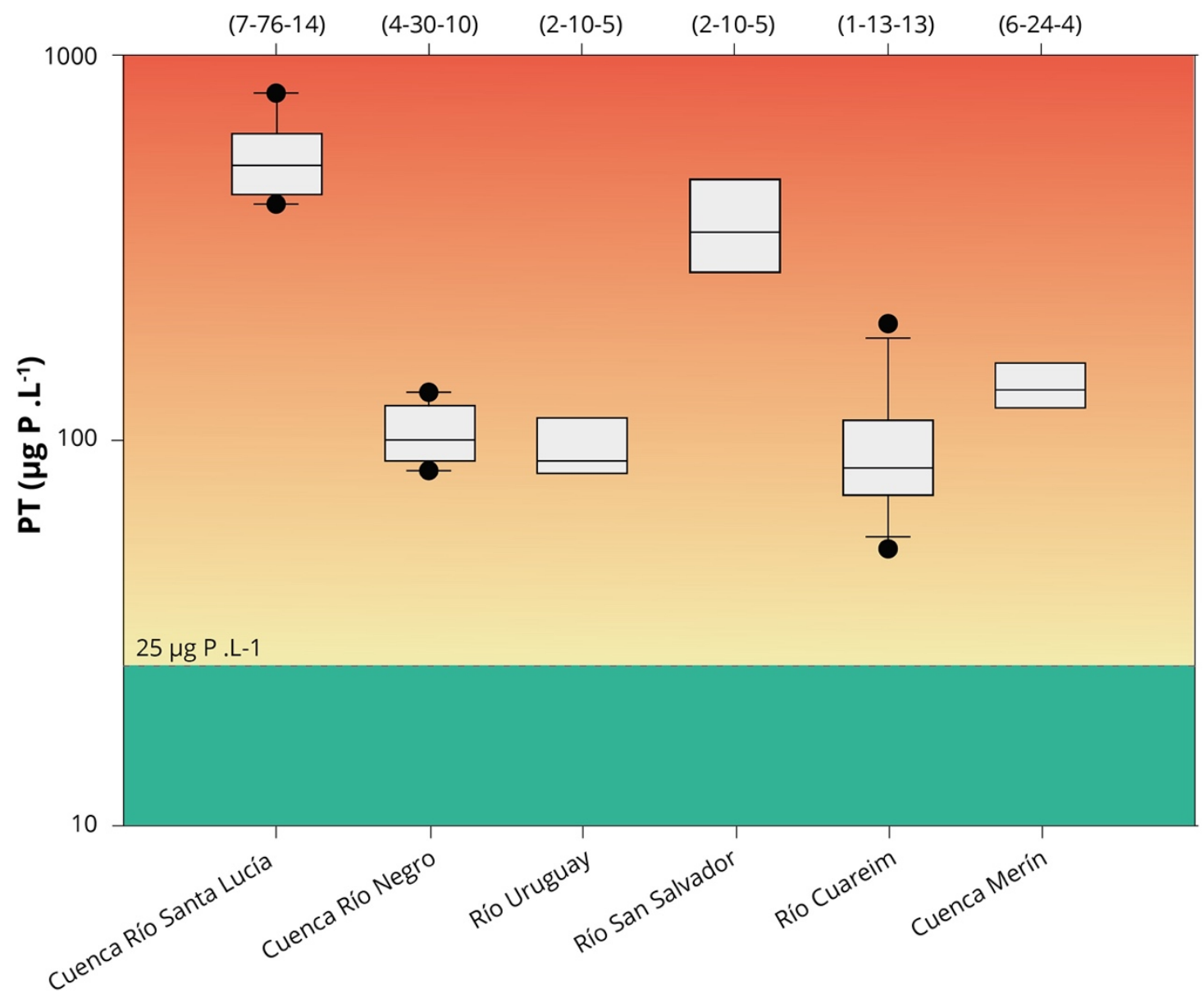

Figura 1. Niveles de fósforo total (PT) para diferentes ecosistemas de aguas corrientes uruguayos de acuerdo con los datos abiertos del Observatorio Ambiental Nacional (Uruguay. Ministerio de Vivienda Ordenamiento Territorial y Medio Ambiente, DINAMA, 2020). Nótese la escala logarítmica en el eje de las ordenadas. La línea sobre el sector verde marca el límite tolerable según la normativa para este tipo de ecosistemas. Cajas: primer, segundo y tercer cuartil; bigotes: primer y noveno decil; puntos negros: outliers. Sobre el eje superior se muestra el número de estaciones de muestreo, el número de datos anuales disponibles, y la mayor extensión en años de cada set de datos. Se dispone de un solo dato por año, el que probablemente sea un promedio de varios muestreos (no se reporta la naturaleza del dato en el Observatorio Ambiental Nacional). No fue posible actualizar los datos en marzo de 2021, ya que el observatorio se encuentra fuera de servicio.

Sin ignorar el rol del nitrógeno como nutriente asociado al proceso de eutrofización (Chorus y Spijkerman, 2021; Paerl, et al., 2016; Schindler, et al., 2016), aquí se pone énfasis en el fósforo por considerarlo de mayor relevancia para la situación actual de Uruguay (Bonilla, et al., 2015; Goyenola, et al., 2020; González-Madina, et al., 2018; Goyenola, 2016). Este diagnóstico puede tomarse 
como válido para gran parte de la región del Río de la Pata, dependiendo de las características biogeoquímicas y los usos del suelo en las cuencas consideradas (Feijoó, et al., 1999; Feijoó y Lombardo, 2007).

\section{Cambio climático y eutrofización}

En los últimos años, se ha alcanzado en la comunidad científica especializada un consenso en cuanto a que el cambio climático potencia la eutrofización y sus síntomas mediante varios procesos (Moss, et al., 2011; Paerl y Huisman, 2008; Li et al., 2021). Se espera que en zonas donde aumenten las precipitaciones ocurra intensificación agrícola y, por el contrario, se esperaría una disminución de la intensidad agrícola en zonas cálidas y mediterráneas debido a una menor disponibilidad de agua (IPCC, 2007; Olesen y Bindi, 2002). Como consecuencia, la creación de embalses probablemente se acelerará en zonas cálidas y mediterráneas (incluso en Uruguay, a partir del impulso dado por la aprobación de la Ley $N^{\circ} 19553$ sobre riego con destino agrario, Uruguay, 2017), con la consecuente potenciación de la eutrofización asociada a los embalses (Illiarze, 2015).

En regiones donde se predice un aumento de la precipitación media, se espera un aumento de la escorrentía superficial y una mayor carga de PT ingresando a los cursos de agua (Jeppesen, et al., 2009; Ockenden, et al., 2017). En consecuencia, incluso manteniendo la extensión e intensidad actual de las actividades productivas en las cuencas, se predice un aumento de hasta un $30 \%$ de las cargas de PT en invierno para el 2050, independientemente de los usos del suelo (Ockenden, et al., 2017). Asimismo, se espera una mayor carga externa de nitrógeno (Jeppesen, et al., 2011), así como de materia orgánica disuelta (Graeber, et al., 2015). Por el contrario, en sitios donde se predice una disminución en las precipitaciones medias, la disminución en la escorrentía (Giorgi y Lionello, 2008) promoverá que las cargas de nutrientes sean menores. Sin embargo, la concentración en el agua puede ser igual o incluso mayor debido a un menor volumen de agua, a una mayor evapotranspiración por el aumento de temperatura (Özen, et al., 2010), así como a una mayor liberación del $\mathrm{P}$ acumulado en los sedimentos hacia la columna de agua provocada en condiciones de anoxia (Jeppesen, et al., 2009).

La mayor temperatura determina también una mayor estratificación térmica, lo que favorece a los organismos que pueden regular su posición en la columna de agua, como varios grupos de cianobacterias (Paerl y Huisman, 2008). Como consecuencia directa e indirecta del calentamiento climático se esperan muchos cambios en las comunidades biológicas (Jeppesen, et al., 2014). En particular, se predicen cambios en la comunidad de peces hacia una dominancia de especies de menor tamaño corporal, y por lo tanto con mayor capacidad reproductiva y mayor metabolismo relativo (Lazzaro, 1997; Meerhoff, et al., 2012; Teixeira de 
Mello, et al., 2009), lo que lleva a una menor biomasa del zooplancton filtrador (Meerhoff, et al., 2007). Como consecuencia, las microalgas y cianobacterias pasarían a estar aún más débilmente controladas por consumo que en el presente.

Todas las aproximaciones comúnmente utilizadas en estudios de efectos del cambio climático predicen, de manera coherente, un aumento en las floraciones de cianobacterias con el calentamiento climático (Jeppesen, et al., 2014; Taranu, et al., 2015), incluso a igualdad de concentraciones de $P$ en el agua (Kosten, et al., 2012; Meerhoff, et al., 2012). Por lo tanto, se espera una menor resistencia y resiliencia de los cuerpos de agua frente a la eutrofización.

\section{SÍNTOMAS DE LA EUTROFIZACIÓN: FLORACIONES CIANOBACTERIANAS}

Las cianobacterias son los organismos capaces de hacer fotosíntesis más antiguos del planeta y pueden desarrollarse en todos los ambientes acuáticos (Whitton, 2012). Muchas especies pueden fijar el $\mathrm{N}_{2}$ atmosférico y habitar ecosistemas donde este nutriente es escaso (Vitousek, et al., 2002), así como captar y/o almacenar P en el citoplasma (Li y Dittrich, 2019).

En las condiciones apropiadas, muchas especies pueden generar floraciones: un crecimiento excesivo de sus poblaciones en relación con su densidad original. Un gran número sintetiza uno o más metabolitos perjudiciales para los animales y particularmente para los mamíferos, incluyendo al ser humano (cianotoxinas) (Testai, et al., 2016). Estos compuestos, de naturaleza química diversa, generan los efectos más negativos de las floraciones (Chorus, et al., 2000).

Estas floraciones son un fenómeno extremadamente frecuente de nuestros ecosistemas acuáticos continentales y costeros (Bonilla, et al., 2015; GonzálezMadina, et al., 2018; Kruk, et al., 2019; Vidal y Britos, 2012). Entre las especies tóxicas que forman floraciones en Uruguay se encuentran las del complejo Microcystis aeruginosa (en adelante CMA), que frecuentemente generan floraciones nocivas a nivel global (Harke, et al., 2016). Estas cianobacterias tienen un estilo de vida colonial y producen floraciones en agua dulce y salobre (Kruk, et al., 2019; Kruk, et al., 2017; Martínez de la Escalera, et al., 2017), y son potencialmente productoras de microcistina, cianotoxina de la cual se han descrito al menos 100 variantes diferentes que pueden causar la muerte de animales y seres humanos (Harke, et al., 2016). Sus toxinas y los genes que codifican las mismas están presentes en poblaciones de CMA de todo el país, especialmente en embalses y playas de ambientes dulceacuícolas, pudiendo llegar hasta el Océano Atlántico (Bonilla, et al., 2015; González-Piana, et al., 2017; Kruk, et al., 2019; Kruk, et al., 2015; Kruk, et al., 2017; Martínez de la Escalera, et al., 2017). Los mecanismos ecológicos y evolutivos que explican el éxito del 
CMA involucran una gran diversidad genética que le permite dominar la comunidad fitoplanctónica bajo diferentes condiciones ambientales (Martínez de la Escalera, et al., 2019). La toxicidad de estas floraciones ha demostrado ser muy alta (Kruk, et al., 2019), revistiendo un elevado riesgo para la salud pública (Vidal, et al., 2017).

En Uruguay ocurren también otros géneros de cianobacterias que causan floraciones tóxicas, entre los que se encuentran Planktothrix, Nodularia, Aphanizomenon, Dolichospermum (Aubriot, et al., 2011; Bonilla, et al., 2015; De León y Yunes, 2001; Haakonsson, et al., 2017; Kozlíková-Zapomělová, et al., 2016; Kruk, et al., 2002; Piccini, et al., 2011; Scasso, et al., 2001; Vidal y Britos, 2012) y Raphidiopsis, incluyendo a $R$. raciborskii (antes denominada Cylindrospermopsis raciborskii (Aguilera, et al., 2018). Especies de estos géneros pueden producir diversas toxinas, tanto microcistinas (Planktothrix y algunas especies de Dolichospermum) como nodularinas (Nodularia), saxitoxinas (Raphidiopsis y especies de Aphanizomenon) y anatoxinas (Dolichospermum).

\section{Efectos de las cianobacterias sobre la salud}

Se estima que el $75 \%$ de las floraciones de cianobacterias en nuestra región son productoras de cientos de variedades de cianotoxinas conocidas que ocurren mezcladas con variedades desconocidas (Giannuzzi, et al., 2017). Los más vulnerables son los niños y adultos inmunodeprimidos, y aquellos con mayor grado de exposición (deportistas náuticos, guardavidas). Las vías de exposición incluyen el contacto directo con el cuerpo (piel, mucosas, oídos, ojos), la aspiración (inhalación), la ingestión accidental de agua o de sedimentos, el consumo de agua o de suplementos alimenticios contaminados y de productos pesqueros provenientes de ambientes con floraciones (peces, mariscos, algas) o regados con agua con cianotoxinas (Giannuzzi, et al., 2017; Testai, et al., 2016), así como el ingreso por vía parenteral por hemodiálisis (Azevedo, et al., 2002). Todas las cianotoxinas afectan piel y mucosas, y son varios los órganos y sistemas blanco que incluso son afectados simultáneamente (sistema inmune, pulmones, riñones, estómago, esófago, colon, y tracto gastrointestinal) (Giannuzzi, et al., 2017). Las microcistinas, además del hígado, afectan todo el tracto gastrointestinal y el sistema inmune, los pulmones y el sistema nervioso (McLellan y Manderville, 2017; Preece, et al., 2017). Los efectos pueden ser agudos (exposición a altas concentraciones) o crónicos (exposición periódica a bajas concentraciones). Ambos efectos pasan muchas veces desapercibidos por parte de especialistas de la salud y de los propios afectados; especialmente los efectos crónicos, ya que presentan síntomas inespecíficos (Giannuzzi, et al., 2017). Varios trabajos demuestran la existencia de efectos en la salud humana como resultado de la exposición crónica a cianotoxinas (Chen, et al., 2009; Li y Dittrich, 2019, McLellan y Manderville, 2017), y por consumo de peces y mariscos de agua dulce o salobre (Poste, et al., 2011), incluso en zonas costeras marinas 
(Gibble, et al., 2016).

En Uruguay se han registrado dos casos confirmados de intoxicación aguda por cianotoxinas, ambos por recreación. En 2007, en el Embalse de Salto Grande, fue afectado un deportista adulto (Giannuzzi, et al., 2011); mientras que, en el verano de 2015, en las playas del este de Montevideo (Carrasco y Malvín) fue afectada una niña de 20 meses. La niña presentó síntomas gastrointestinales y fallas graves en el hígado que requirieron un trasplante por hepatitis fulminante por cianotoxinas (Vidal, et al., 2017). En ambos casos, los diagnósticos primarios fueron equivocados (estrés y hepatitis autoinmune, respectivamente), lo que destaca la invisibilidad del riesgo de exposición a floraciones y la falta de conexión entre la información ambiental y el sistema de atención en salud. Uruguay no realiza registro de casos de afectación de salud por exposición a cianobacterias en ninguna de sus formas. Además, las cianotoxinas no están incluidas de forma explícita en el Reglamento Bromatológico Nacional, incluyendo recursos pesqueros, lo cual dificulta no sólo la concientización de la población, sino también el conocimiento de su impacto en la salud pública. A nivel de producción animal, se comparte la preocupación a partir de varios casos de muerte de animales que abrevaron en aguas con floraciones (Bianchi, 2020).

\section{¿CONTROL DE LA EUTROFIZACIÓN O CONTROL DE SUS SÍNTOMAS?}

Esperar a que los síntomas de la eutrofización se expresen en toda su intensidad es una mala opción, ya que la rehabilitación o restauración es muy compleja y costosa, entre otras cosas, porque el proceso presenta una lógica acumulativa y resiliente. Aun en los casos en que se ha logrado un descenso de los niveles de nutrientes hasta los niveles anteriores a la ocurrencia de síntomas notorios, la problemática suele persistir debido a la carga interna de nutrientes (Søndergaard, et al., 2003), los cambios ocurridos en la trama trófica (Jeppesen, et al., 2014) y a que las cianobacterias generan condiciones que facilitan su perpetuación (Ger, et al., 2014; Li, et al., 2021). Si se pretende observar resultados en un período acotado (años, lustros o décadas), generalmente no resulta suficiente controlar las fuentes de nutrientes a escala de la cuenca hidrográfica o disminuir su movilización, transporte y/o llegada a los ecosistemas acuáticos (Haygarth, et al., 2005) para alcanzar umbrales de nutrientes mucho menores a los que promovieron los síntomas (Schindler, 2006). Además, debe considerarse que los umbrales de nutrientes para recuperar los ecosistemas deberán ser aún menores en el futuro cercano, dado el efecto sinérgico de la eutrofización con el cambio climático.

Luego de revertir las causas, suele ser necesario manipular comunidades biológicas clave para que se recuperen procesos ecológicos asociados a un estado no eutrófico (Moss, et al., 1996). Estas medidas incluyen reestablecer macrófitas (particularmente especies enraizadas), o controlar su crecimiento 
excesivo (Basset, et al., 2014), o modificar la trama trófica para aumentar la presión de consumo sobre el fitoplancton a través de técnicas denominadas biomanipulación. En este caso, la reintroducción de especies de peces potencialmente piscívoras $y / o$ la remoción de peces potencialmente zooplanctívoros o bentívoros, repercuten positivamente sobre la calidad de agua (Jeppesen, et al., 2012). Estas medidas requieren múltiples intervenciones si no se controla la carga externa e interna de nutrientes y su éxito varía enormemente según el contexto climático y biogeográfico (Jeppesen, et al., 2007, Jeppesen, et al., 2012).

Bases conceptuales para la reducción de las pérdidas difusas de fósforo desde suelos bajo uso agropecuario

En el control y manejo de la eutrofización, los mayores avances a nivel internacional se observan en relación con los aportes puntuales, debido a las tecnologías disponibles y el rol de los Estados en la provisión de servicios básicos de agua y saneamiento. Los grandes desafíos permanecen en el campo del control del aporte difuso.

Las pérdidas difusas de $\mathrm{P}$ desde suelos bajo producción agropecuaria convencional se asocian al transporte por escorrentía superficial. De acuerdo con el paradigma vigente hasta unos pocos años atrás, cabría esperar que el $P$ se transportase mayormente en forma particulada (Correll, 1998; Sharpley, et al., 2001). En consecuencia, si se controlara la erosión del suelo, se podrían mantener estas pérdidas difusas en niveles aceptables. Sin embargo, resultados más recientes a nivel internacional indican que bajo siembra directa donde prima la aplicación de $\mathrm{P}$ en superficie, las pérdidas solubles son muy altas (Jarvie, et al., 2017). Esto se condice con hallazgos a nivel nacional sobre las fracciones de $P$ dominantes en arroyos de cuencas con distintos usos productivos (Aubriot, et al., 2017; Chalar, et al., 2017; Goyenola,2016, Goyenola et al., 2015). Por tanto, solo el control de la erosión no es suficiente para mantener estas pérdidas dentro de límites compatibles con una buena calidad del agua (Jarvie, et al., 2017).

Una parte de las pérdidas de $\mathrm{P}$ soluble se debe a la pérdida directa del fertilizante, que permanece soluble durante unos meses post-aplicación. De acuerdo con datos empíricos, estas pérdidas pueden llegar hasta el $5 \%$ de la dosis de $\mathrm{P}$ aplicada, aunque los valores más probables oscilan entre el $1-2 \%$ (Perdomo, et al., 2015). Por otra parte, la aplicación continua y durante muchos años de $P$ en superficie produce una estratificación vertical del $P$ fácilmente disponible (denominado P lábil) en zonas bajo siembra directa. En Uruguay se ha reportado aumento del $\mathrm{P}$ lábil en superficie (medido como PBray1), entre 4 y 8 veces de lo que resultaría si el $\mathrm{P}$ se distribuyera homogéneamente en los primeros $15 \mathrm{~cm}$ de suelo, tal como ocurría antes de que se adoptase la siembra 
directa en Uruguay a partir de 1999 (Perdomo, et al., 2015). Estudios realizados en unidades de manejo bajo siembra directa en predios lecheros de la cuenca del Río Santa Lucía mostraron que la concentración de P soluble extraído con agua aumentó linealmente con la concentración de $P$ lábil en muestras superficiales de suelo (Bray $1 ; 2,5 \mathrm{~cm})(\mathrm{r} 2$ Pearson $=0,73 ; p<0,001 ; n=38$, Perdomo, et al., 2015). Por lo tanto, aunque la práctica de aplicación superficial de $\mathrm{P}$ cesara, las pérdidas incrementadas de $\mathrm{P}$ soluble desde los suelos agrícolas continuarían durante algunas décadas o incluso cientos de años, según distintas estimaciones teóricas.

\section{Cambios y desafíos en llos sistemas de gestión de la eutrofización}

Uruguay presenta cambios muy importantes en el análisis y la gestión de la eutrofización vinculados a las transformaciones del sistema de gobernanza del agua. Desde inicios de los años 2000, el país ha navegado desde un modelo fragmentado-jerárquico (conocido como "comando-control") hacia un paradigma de gestión integrada con diversas estructuras puente que promueven la interacción entre actores gubernamentales, académicos y la sociedad civil. La inercia del sistema es muy grande por múltiples factores, pero la eutrofización ha actuado como un factor externo acelerando las transformaciones (Mazzeo, et al., 2019; Trimble, et al., 2020; Trimble y Mazzeo, 2020).

\section{DISCUSIÓN Y CONCLUSIONES}

A pesar de que se contaba con diagnósticos técnicos concluyentes, las crisis generadas por la afectación del agua potable entre 2013 y 2015 fueron el factor que posibilitó la aprobación de planes de acción tendientes a proteger la calidad ambiental y la disponibilidad de las fuentes de agua en Uruguay. Estos eventos dispararon también multimillonarias inversiones para mejorar tanto los sistemas de potabilización como de tratamiento de aguas residuales.

En lo que va de este siglo, el sistema productivo agrícola-ganadero ha aumentado su intensidad e incrementado su eficiencia (Uruguay. Ministerio de Ganadería Agricultura y Pesca, DIEA, 2019), incorporando prácticas de conservación de suelos que mejoraron la sustentabilidad ambiental del sector (Ley No 15.239 y decretos reglamentarios, Uruguay 1981, 1990, 2004 y 2008). No obstante, las cargas de nutrientes exportadas hacia los ecosistemas acuáticos han aumentado significativamente. La prueba más concluyente es que la eutrofización se ha instalado como un problema ambiental generalizado y de gran magnitud en Uruguay, habiendo provocado consecuencias negativas y a gran escala para la potabilización para consumo humano, las actividades recreativas y el turismo, además de para la biodiversidad acuática. 
La escala de las floraciones cianobacterianas potencialmente tóxicas, ocurridas durante la última década, muestra que su magnitud y frecuencia están en pleno aumento, lo que concuerda con: a) la elevada biodisponibilidad de $\mathrm{P}$, dada por las altas concentraciones y por el predominio de la fracción disuelta; b) el enriquecimiento desproporcionado del $\mathrm{P}$ respecto al $\mathrm{N}$ con la intensidad productiva actual, que favorece a las especies capaces de fijar $\mathrm{N}_{2(\mathrm{~g})}$ atmosférico; c) la baja capacidad de control natural por consumo (herbivoría), dada por las características de las redes tróficas subtropicales, y d) los efectos predichos del cambio climático. En este contexto, encontrar el compromiso adecuado entre la producción de alimentos y la generación de divisas, por un lado, y la conservación de los ecosistemas y disponer de agua adecuada para potabilizar, por otro, es uno de los mayores retos que enfrenta nuestra sociedad actualmente (Meerhoff y Bernardi, 2018).

Aunque el tema ha sido profusamente discutido en ámbitos técnicos, e incluso en medios de prensa, existe suficiente evidencia científica para asegurar que en Uruguay la eutrofización es una externalidad causada por la actividad agrícola-ganadera (Chalar, et al., 2017; Goyenola, 2016; Goyenola, et al., 2015; Goyenola, et al., 2020) y las carencias de los sistemas de tratamiento de efluentes (Olano, et al., 2019). Cualquiera de los dos tipos de aportes por sí solos puede generar condiciones de eutrofización en el Uruguay actual, por lo tanto, preguntarse cuál de los aportes es el más importante (difuso vs. puntual) no es pertinente cuando el objetivo es controlar la problemática a escala país. La reciente intensificación de la eutrofización deriva de factores como la baja eficiencia en el uso de nutrientes, y los efectos no intencionales de las prácticas productivas conservacionistas del suelo que favorecen la movilización y el transporte del fósforo soluble por escorrentía superficial (Chalar, et al., 2017; Uruguay. Ministerio de Ganadería Agricultura y Pesca, DIEA, 2019; Goyenola, 2016; Goyenola, et al., 2015; Goyenola, et al., 2020; Jarvie, et al., 2017; Perdomo, et al., 2015). A lo anterior se le suma un escenario de cambios en los regímenes de precipitación (Bidegain, et al., 2013), que afecta la magnitud de los procesos biogeoquímicos a escala del paisaje (Jeppesen, et al., 2009).

Para minimizar la carga de fósforo exportado por las actividades productivas es necesario ajustar las aplicaciones de fertilizantes a dosis racionales (dosis recomendadas por cultivo según niveles de nutrientes en suelo y planta), e incorporar el fertilizante fosfatado por debajo de la zona de mezclado del suelo, es decir, la capa superficial de menos de $3 \mathrm{~cm}$ donde el agua de escurrimiento interacciona con el suelo y con el $\mathrm{P}$ soluble proveniente del fertilizante (Jarvie, et al., 2017; Smith, et al., 2015). Debe considerarse la implementación de la inversión esporádica del suelo con arado de reja y vertedera (Baker, et al., 2017; Smith, et al., 2017) para eliminar la estratificación vertical del fósforo y la saturación en superficie, así como para disminuir la compactación y aumentar la 
infiltración. La aplicación combinada de estas medidas debería sumarse al mantenimiento de las medidas para el control de la erosión del suelo.

En los casos donde la acumulación de $\mathrm{P}$ lábil se extiende en profundidad en el suelo, la inversión del suelo ya no sería efectiva; por lo que, además de suspender la fertilización, habría que promover la extracción paulatina del P lábil mediante cultivos ("catch crops"; Eghball, et al., 2003). Aunque los estudios a escala de campo aún son incipientes, otras medidas para reducir el $\mathrm{P}$ lábil incluyen la aplicación de productos químicos al suelo para transformarlo rápidamente a formas menos biodisponibles (Penn, et al., 2012), o eventualmente, combinar estas dos últimas prácticas (Schärer, et al., 2007).

El reciclaje de nutrientes intrapredial, por ejemplo, mediante la utilización de abono de estiércol, se realiza habitualmente en otros países (Jeppesen, et al., 2011). Esta estrategia es particularmente relevante dado que aún en condiciones con mínimo impacto relativo, como la actividad ganadera extensiva tradicional en los pastizales de la región del Río de la Plata (Modernel, et al., 2016), los niveles de PT en los cursos aguas abajo exceden con frecuencia tanto el estándar (exigente) previsto en la normativa uruguaya, como límites aceptables desde un punto de vista académico (más flexibles) (Carlson, 1977, Lamparelli, 2004). Los sistemas productivos que durante muchos años mantienen balances de $\mathrm{P}$ positivos inexorablemente generan problemas de eutrofización de las aguas (Powers, et al., 2016). Por ello, todas las medidas descritas son válidas en el corto y mediano plazo. La solución a largo plazo es, incluso dentro del paradigma de producción convencional, transitar hacia sistemas productivos con balances de $P$ neutros o negativos (Peterson, et al., 2017; Reid, et al., 2019; Ruane, et al., 2014). Dada la magnitud de la eutrofización, para lograr revertir la tendencia a mediano plazo (e inclusive para lograr mantener la calidad del agua en los niveles actuales de impacto), es imprescindible aplicar medidas a escala de cuenca hidrográfica que aumenten la retención de nutrientes. En este marco, la conservación y recuperación de humedales y zonas riparias cumple un rol fundamental (Stutter, et al., 2012).

Para ajustar los sistemas productivos y promover la resiliencia natural de los ecosistemas, es clave la generación de conocimiento original específico y la innovación ajustada a la dinámica de nuestros ecosistemas y así contribuir a la toma de decisiones informada y basada en el mejor conocimiento científico disponible (Tabla 2) (Sharpley, et al., 2015).

En Uruguay, la ausencia de sistemas de monitoreo suficientes a escala país y de evaluaciones sólidas de los impactos generados por las transformaciones productivas (inclusive sobre la salud pública) limitan, tanto el diseño de medidas de gestión adecuadas, como el manejo de la incertidumbre inherente a estas 
problemáticas y la capacidad de aprendizaje (generalmente asociados al aprender haciendo) (Kleinman, et al., 2015; Pahl-Wostl, 2015). Con excepción del registro de caudales, los sistemas de monitoreo en Uruguay no son de larga duración, no están diseñados siguiendo un enfoque integral (por ejemplo, el monitoreo de calidad suele no considerar cantidad del agua, y viceversa), carecen de cobertura en grandes sectores del territorio, se encuentran preferentemente enfocados en ecosistemas de aguas corrientes de alto orden (grandes ríos), no incluyen ecosistemas de referencia (cuerpos de agua con el menor impacto posible), y no permiten extrapolar información a ecosistemas no monitoreados. Además, la evaluación de la calidad del agua se basa casi exclusivamente en propiedades físico-químicas, sin considerar bioindicadores 0 atributos ecosistémicos (Mazzeo, et al., 2019). Estos aspectos están incluidos desde hace décadas en normativas internacionales, como la Directiva Marco del Agua de la Unión Europea, 2000/60/CE (Unión Europea, 2000).

La generación de conocimiento es un paso ineludible (Tabla 2). No obstante, la gestión de esta problemática es un desafío fundamentalmente organizacional, que requiere modificaciones importantes de un sistema de gobernanza centralista y que, aunque supera la fragmentación en el análisis y toma de decisión, no lo hace en el campo de su aplicación, control y fiscalización. En este sentido, la creación del Ministerio de Ambiente en 2020 genera una oportunidad y un desafío para mantener y profundizar los pasos positivos y para realizar los cambios necesarios para enfrentar la eutrofización en el Uruguay. Estas transformaciones deberían ser acompañadas por procesos de descentralización y de espacios multiactorales efectivos que promuevan la interacción interinstitucional e internivel, y el intercambio entre los dominios disciplinares y sistemas de conocimiento. 
Tabla 2. Algunos aspectos clave en los que el Uruguay podría y debería avanzar en los próximos años con el objetivo de disminuir la eutrofización y sus impactos.

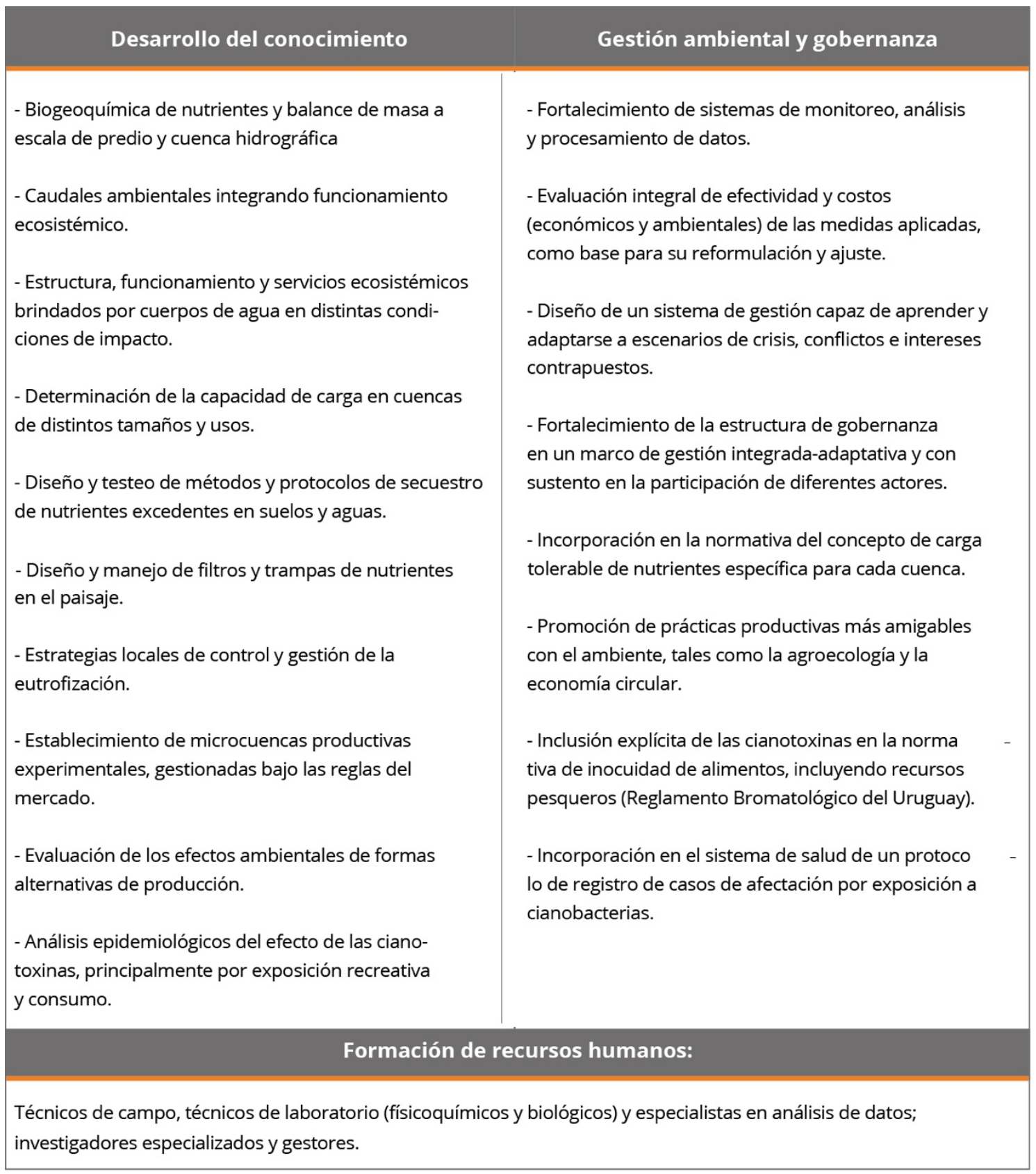

\section{AGRADECIMIENTOS}

Este manuscrito integra aportes realizados por un grupo de académicos y gestores ambientales en la mesa redonda organizada en el $\mathrm{X}$ Congreso de la Red Latinoamericana de Ciencias Ambientales en marzo de 2020 (Maldonado, Uruguay). Los autores agradecen muy especialmente a la Red y a los organizadores del X Congreso. Queremos también agradecer a Diego Michelini 
y Micaela Trimble por los aportes realizados. Goyenola, Kruk, Mazzeo, Meerhoff, Piccini y Perdomo son apoyados por el SNI-ANII. Meerhoff y Goyenola fueron apoyados por el Consejo Danés de Investigación Independiente, ANII-FCE (20092749), Meerhoff contó con el apoyo del premio nacional L'Oreal-UNESCO para las Mujeres en la Ciencia con apoyo de DICyT y de CSIC, Artículo 22019 proyecto: De la Naturaleza a tu mesa: huella ambiental, social y económica de la producción de alimentos en Uruguay. Mazzeo por el Programa de Grupos de InvestigaciónCSIC y Proyecto Governagua. Perdomo contó con el apoyo del Fondo María Viñas FMV_3_2011_1_7088, European Union-INCOCT-2006-032387, INIA-FPTA 301 2013, beca ANII Posgrados 2012_1_8898, MGAP-DACC, CONICYT Udelar, UdelaR (becas posgrado y visita científica). Piccini contó con el apoyo del proyecto FCE_1_2019_1_156308 (ANII). En el artículo se han incorporado contribuciones en el área de la gobernanza del agua en tres proyectos en pleno desarrollo: "Proceso de construcción de la gestión integrada de recursos hídricos en Uruguay, estado de situación y controversias", financiado por el Global Water Parternship (GWP); "Transformando la gobernanza del agua en América del Sur: de la reacción a la adaptación y la anticipación", financiado por el Instituto Interamericano para la Investigación del Cambio Global (IAI) SGP-HW 056; y "Bases para el diseño de un sistema nacional de monitoreo de recursos hídricos desde la perspectiva de cuencas virtuales" (ANII, FSDA 120181 154610).

\section{REFERENCIAS}

Aguilera, A., Gómez, E.B., Kaštovský, J., Echenique, R.O. y Salerno, G.L., 2018. The polyphasic analysis of two native Raphidiopsis isolates supports the unification of the genera Raphidiopsis and Cylindrospermopsis (Nostocales, Cyanobacteria). En: Phycologia, 57(2), pp.130-146. https://doi.org/10.2216/17-2.1

Aubriot, L., Bonilla, S. y Falkner, G., 2011. Adaptive phosphate uptake behaviour of phytoplankton to environmental phosphate fluctuations. En: FEMS Microbiology Ecology, 77, pp.1-16. https://doi.org/10.1111/j.1574-6941.2011.01078.x

Aubriot, L., Delbene, L., Haakonsson, S., Somma, A., Hirsch, F. y Bonilla, S., 2017. Evolución de la eutrofización en el Río Santa Lucía: influencia de la intensificación productiva y perspectivas. En: INNOTEC, 14, pp.7-16. https://doi.org/10.26461/14.04

Azevedo, S.M.F.O., Carmichael, W.W., Jochimsen, E.M., Rinehart, K.L., Lau, S., Shaw, G.R. y Eaglesham, G.K., 2002. Human intoxication by microcystins during renal dialysis treatment in Caruaru/Brazil. En: Toxicology, 181/182, pp.441-446. https://doi.org/10.1016/S0300-483X(02)00491-2 
Baker, D.B., Johnson, L.T., Confesor, R.B. y Crumrine, J.P., 2017. Vertical stratification of soil phosphorus as a concern for dissolved phosphorus runoff in the lake Erie Basin. En: Journal of Environmental Quality, 46, pp.1287-1295. https://doi.org/10.2134/jeq2016.09.0337

Barreto, P., Ernst, O., Bidegáin, P.M. y Perdomo, C., 2014. Cuantificación de la pérdida de fósforo por escorrentía en diferentes rotaciones de suelos de Uruguay [En línea]. En: Congreso Uruguayo de Suelos. Colonia, Uruguay (6-8 de agosto de 2014). Colonia: [s.n.]. [Consulta: 01 de agosto de 2020]. Disponible en: http://www.suelos.com.uy/pdf/58.pdf

Basset, A., Baxter, J.M. y Daborn, G.R., 2014. Report Laguna del Diario. Rehabilitation strategy of the lagoon ecological status [En línea]. Montevideo: SMRU Marine. [Consulta: 01 de agosto de 2020]. Disponible en: http://www.maldonado.gub.uy/files/17162_2ec0b8_REPORTE-FINALINGLES.pdf

Bianchi, C., 2020. Uriarte: Este gobierno ignoró a los productores rurales. Hay que escucharlos. [En línea]. Montevideo: Montevideo Portal. [Consulta: 09 de agosto de 2020]. Disponible en: https://www.montevideo.com.uy/Noticias/Uriarte--Este-gobierno-ignoroa-los-productores-rurales- Hay-que-escucharlos--uc740016

Bidegain, M., Crisci, C., Del Puerto, L., Inda, H., Mazzeo, N., Taks, J. y Terra, R., 2013. Clima de cambios, nuevos desafíos de adaptación en Uruguay. Vol. 1 Variabilidad climática de importancia para el sector productivo. FAOMGAP. TCP URU/3302 [En linea]. Montevideo: FAO. [Consulta: 09 de agosto de 2020]. Disponible en: http://www.fao.org/3/as253s/as253s.pdf

Bonilla, S., Haakonsson, S., Somma, A., Gravier, A., Britos, A., Vidal, L., De León, L., Brena, B., Pírez, M., Piccini, C., Martínez De la Escalera, G., Chalar, G., González-Piana, M., Martigani, F. y Aubriot, L., 2015. Cianobacterias y cianotoxinas en ecosistemas límnicos de Uruguay. En: INNOTEC, 10, pp.922.

Carlson, R.E., 1977. A Trophic State Index for Lakes. En: Limnology and Oceanography, 22, pp.361-369. https://doi.org/10.4319/lo.1977.22.2.0361 
Chalar, G., Garcia-Pesenti, P., Silva-Pablo, M., Perdomo, C., Olivero, V. y Arocena, R., 2017. Weighting the impacts to stream water quality in small basins devoted to forage crops, dairy and beef cow production. En: LimnologicaEcology and Management of Inland Waters, 65, pp.76-84. https://doi.org/10.1016/j.limno.2017.06.002

Chen, J., Xie, P., Li, L. y Xu, J., 2009. First identification of the hepatotoxic microcystins in the serum of a chronically exposed human population together with indication of hepatocellular damage. En: Toxicological Sciences, 108, pp.81-89. https://doi.org/10.1093/toxsci/kfp009

Chorus, I., Falconer, I.R., Salas, H.J. y Bartram, J., 2000. Health risks caused by freshwater cyanobacteria in recreational waters. En: Journal of Toxicology and Environmental Health, Part B: Critical Reviews, 3, pp.323-347. https://doi.org/10.1080/109374000436364

Chorus, I. y Spijkerman, E., 2021. What Colin Reynolds could tell us about nutrient limitation, $\mathrm{N}: \mathrm{P}$ ratios and eutrophication control. En: Hydrobiologia, 848, pp.95-111. https://doi.org/10.1007/s10750-02004377-w

CLAES, PNUMA y DINAMA, 2008. GEO Uruguay. Informe del estado del ambiente. Montevideo: Mosca. 350 pp.

Cordell, D., Drangert, J.O. y White, S., 2009. The story of phosphorus: global food security and food for thought. En: Global Environmental Change, 19, pp.292-305. https://doi.org/10.1016/j.gloenvcha.2008.10.009

Correll, D.L., 1998. The role of phosphorus in the eutrophication of receiving waters: a review. En: Journal of Environmental Quality, 27, pp.261-266. https://doi.org/10.2134/jeq1998.00472425002700020004x

De León, L. y Yunes, J.S., 2001. First report of a microcystin-containing bloom of the cyanobacterium microcystis aeruginosa in the La Plata River, South America. En: Enviromental Toxicology, 16, pp.110-112. https://doi.org/10.1002/15227278(2001)16:1<110::AID-TOX1012>3.0.CO;2Z

Dodds, W.K., 2006. Eutrophication and trophic state in rivers and streams. En: Limnology and Oceanography, 51, pp.671-680. https://doi.org/10.4319/lo.2006.51.1_part_2.0671 
Dolman, A.M., Rücker, J., Pick, F.R., Fastner, J., Rohrlack, T., Mischke, U. y Wiedner, C. 2012. Cyanobacteria and Cyanotoxins: The Influence of Nitrogen versus Phosphorus. En: PLoS ONE, 7, e38757. https://doi.org/10.1371/journal.pone.0038757

Eghball, B., Shanahan, J.F., Varvel, G.E. y Gilley, J.E., 2003. Reduction of high soil test phosphorus by corn and soybean varieties. En: Agronomy Journal, 95, pp.1233-1239. https://doi.org/10.2134/agronj2003.1233

Feijoó, C.S., Giorgi, A., García, M.E. y Momo, F., 1999. Temporal and spatial variability in streams of a pampean basin. En: Hydrobiologia, 394, pp.41-52. https://doi.org/10.1023/A:1003583418401

Feijoó, C.S. y Lombardo, R.J. 2007. Baseline water quality and macrophyte assemblages in Pampean streams: A regional approach. En: Water Research, 41, pp.1399-1410. https://doi.org/10.1016/j.watres.2006.08.026

Fowler, D., Coyle, M., Skiba, U., Sutton, M.A., Cape, J.N., Reis, S., Sheppard, L.J., Jenkins, A., Grizzetti, B., Galloway, J.N., Vitousek, P., Leach, A., Bouwman, A.F., Butterbach-Bahl, K., Dentener, F., Stevenson, D., Amann, M. y Voss, M., 2013. The global nitrogen cycle in the twenty-first century. En: Philosophical Transactions of the Royal Society of London B: Biological Sciences, 368(1621). https://doi.org/10.1098/rstb.2013.0164

García-Rodríguez, F., Mazzeo, N., Sprechmann, P., Metzeltin, D., Sosa, F., Treutler, H.C., Renom, M., Scharf, B. y Gaucher, C., 2002. Paleolimnological assessment of human impacts in Lake Blanca, SE Uruguay. En: Journal of Paleolimnology, 28, pp.457-468. https://doi.org/10.1023/A:1021616811341

Gazzano, I., Achkar, M. y Díaz, I., 2019. Agricultural transformations in the southern cone of Latin America: agricultural intensification and decrease of the aboveground net primary production, Uruguay's case. En: Sustainability, 11, pp.7011. https://doi.org/10.3390/su11247011

Ger, K.A., L.A. Hansson y M. Lürling, 2014. Understanding cyanobacteria zooplankton interactions in a more eutrophic world. En: Freshwater Biology, 59, 1783-1798. doi:10.1111/fwb.12393

Giannuzzi, L., Petcheneshsky, T. y Hansen, M., 2017. Cianobacterias como determinantes ambientales de la salud. Buenos Aires: Ministerio de Salud de la Nación. 
Giannuzzi, L., Sedan, D., Echenique, R. y Andrinolo, D., 2011. An acute case of intoxication with cyanobacteria and cyanotoxins in recreational water in Salto Grande Dam, Argentina. En: Marine Drugs, 9, pp.2164-2175. https://doi.org/10.3390/md9112164

Gibble, C.M., Peacock, M.B. y Kudela, R.M., 2016. Evidence of freshwater algal toxins in marine shellfish: implications for human and aquatic health. En: Harmful Algae, 59, pp.59-66. https://doi.org/10.1016/j.hal.2016.09.007

Giorgi, F. y Lionello, P., 2008. Climate change projections for the Mediterranean region. En: Global and Planetary Change, 63, pp.9-104. https://doi.org/10.1016/j.gloplacha.2007.09.005

González-Madina, L., Pacheco, J.P., Yema, L., Tezanos, P.D., Levrini, P., Clemente, J., Crisci, C., Lagomarsino, J.J., Méndez, G., Fosalba, C., Goyenola, G. y Mazzeo, N., 2018. Drivers of cyanobacteria dominance, composition and nitrogen fixing behavior in a shallow lake with alternative regimes in time and space, Laguna del Sauce (Maldonado, Uruguay). En: Hydrobiologia, 289, pp.61-76. https://doi.org/10.1007/s10750-018-3628-6

González-Piana, M., Fabián, D., Piccardo, A. y Chalar, G., 2017. Dynamics of total microcystin LR concentration in three subtropical hydroelectric generation reservoirs in Uruguay, South America. En: Bulletin of Environmental Contamination and Toxicology, 99, pp.488-492. https://doi.org/10.1007/s00128-017-2195-2

Goyenola, G., 2016. Efectos de la intensidad productiva agrícola sobre la dinámica de macronutrientes en arroyos de cabecera: Una evaluación bajo condiciones climáticas/hidrológicas contrastantes. Montevideo: UdelaR, Facultad de Ciencias. (Tesis de doctorado).

Goyenola, G., Meerhoff, M., Teixeira-De Mello, F., González-Bergonzoni, I., Graeber, D., Fosalba, C., Vidal, N., Mazzeo, N., Ovesen, N. B., Jeppesen, E. y Kronvang, B., 2015. Monitoring strategies of stream phosphorus under contrasting climate-driven flow regimes. En: Hydrology and Earth System Sciences, 19, pp.4099-4111. https://doi.org/10.5194/hess-19-4099-2015

Goyenola, G., Vidal, N., Acevedo, S., Cabrera, S., Fosalba, C., Teixeira-De Mello, F., Calvo, C., Tesitore, G., Gaucher, L., Iglesias, C., López-Rodríguez, A., Burwood, M., Corrales, N., Olsson, D., Levrini, P., Pacheco, J. P., Capuccio, L. y Urtado, L., 2017. Sistemas acuáticos canarios. Estado del conocimiento y gestión ambiental. Informe ambiental estratégico. Canelones: Centro Universitario Regional Este, Universidad de la República, Comuna Canaria. 
Goyenola, G., Graeber. D., Meerhoff, M., Jeppesen, E., Mello, F.D., Vidal, N., Fosalba, C., Ovesen, N.B., Gelbrecht, J., Mazzeo, N. y Kronvang, B., 2020. Influence of farming intensity and climate on lowland stream nitrogen. En: Water, 12(4), pp.1021. https://doi.org/10.3390/w12041021

Graeber, D., Boëchat, I.G., Encina-Montoya, F., Esse, C., Gelbrecht, J., Goyenola, G., Gücker, B., Heinz, M., Kronvang, B., Meerhoff, M., Nimptsch, J., Pusch, M. T., Silva, R.C.S., Von Schiller, D. y Zwirnmann, E., 2015. Global effects of agriculture on fluvial dissolved organic matter. En: Scientific Reports, 5, pp.16328. https://doi.org/10.1038/srep16328

Haakonsson, S., Rodríguez-Gallego, L., Somma, A. y Bonilla, S., 2017. Temperature and precipitation shape the distribution of harmful cyanobacteria in subtropical lotic and lentic ecosystems. En: Science of The Total Environment, 609, pp.1132-1139.

https://doi.org/10.1016/j.scitotenv.2017.07.067

Harke, M.J., Steffen, M.M., Gobler, C.J., Otten, T.G., Wilhelm, S.W., Wood, S.A. y Paerl, H.W., 2016. A review of the global ecology, genomics, and biogeography of the toxic cyanobacterium, Microcystis spp. En: Harmful Algae, 54, pp.4-20. https://doi.org/10.1016/j.hal.2015.12.007

Haygarth, P.M., Condron, L.M., Heathwaite, A.L., Turner, B.L. y Harris, G.P., 2005. The phosphorus transfer continuum: linking source to impact with an interdisciplinary and multi-scaled approach. En: Science of the Total Environment, 344, pp.5-14. https://doi.org/10.1016/j.scitotenv.2005.02.001

Illiarze, M., 2015. Embalses como incubadoras de cianobacterias: efecto de la residencia del agua y de la disponibilidad de nutrientes. Montevideo: UdelaR, Facultad de Ciencias. (Tesis de grado).

Inda, H., García-Rodríguez, F., Stutz, S., Lopes Figueira, R., Alves De Lima Ferreira, P. y Mazzeo, N., 2016. Discriminating between natural and human-induced shifts in a shallow coastal lagoon: A multidisciplinary approach. En: Anthropocene, 16, pp.1-15. https://doi.org/10.1016/j.ancene.2016.09.003

IPCC, 2007. Summary for policymakers. En: Solomon, S., Qin, D., Manning, M., Enhen, Z., Marquis, M., Averyt, K.B., Tignor, M. y Miller, H.L., eds. Climate change 2007: the physical science basis. Contribution of working group I to the fourth assessment report of the intergovernmental panel on climate change. Nueva York: Cambridge University Press. 
Jarvie, H.P., Johnson, L.T., Sharpley, A.N., Smith, D.R., Baker, D.B., Bruulsema,

T.W. y Confesor, R., 2017. Increased soluble phosphorus loads to lake erie: unintended consequences of conservation practices? En: Journal of Environmental Quality, 46, pp.123-132. https://doi.org/10.2134/jeq2016.07.0248

Jeppesen, E., Kronvang, B., Meerhoff, M., Søndergaard, M., Hansen, K.M., Andersen, H.E., Lauridsen, T.L., Beklioglu, M. y Olesen, A.O.J.E., 2009. Climate change effects on runoff, phosphorus loading and lake ecological state, and potential adaptations. En:Journal of Environmental Quality, 38, pp.1930-1941. https://doi.org/10.2134/jeq2008.0113

Jeppesen, E., Kronvang, B., Olesen, J., Audet, J., Søndergaard, M., Hoffmann, C., Andersen, H., Lauridsen, T., Liboriussen, L., Larsen, S., Beklioglu, M., Meerhoff, M., Özen, A. y Özkan, K., 2011. Climate change effects on nitrogen loading from cultivated catchments in Europe: implications for nitrogen retention, ecological state of lakes and adaptation. En: Hydrobiologia, 663, pp.1-21. https://doi.org/10.1007/s10750-010-0547-6

Jeppesen, E., Meerhoff, M., Davidson, T.A., Trolle, D., Søndergaard, M., Lauridsen, T.L., Beklioglu, M., Brucet, S., Volta, P., González-Bergonzoni, I. y Nielsen, A., 2014. Climate change impacts on lakes: an integrated ecological perspective based on a multi-faceted approach, with special focus on shallow lakes. En: Journal of Limnology, 73, pp.84-107. https://doi.org/10.4081/jlimnol.2014.844

Jeppesen, E., Meerhoff, M., Jacobsen, B.A., Hansen, R.S., Søndengaard, M., Jensen, J.P., Lauridsen, T., Mazzeo, N. y Branco, C.C., 2007. Restoration of shallow lakes by nutrient control and biomanipulation the successful strategy varies with lake size and climate. En: Hydrobiologia, 581, pp.269285.

Jeppesen E., Søndergaard, M., Lauridsen, T.L., Davidson, T.A., Liu, Z., Mazzeo, N., Trochine, C., Ozkan, K., Jensen, H.S., Trolle, D., Landkildehus, F., Starling, F., Larsen, S.E., Lazzaro, X. y Meerhoff, M., 2012. Biomanipulation as a restoration tool to combat eutrophication - recent advances and future challenges.En: Advances in Ecological Research, 47, pp.411-488. https://doi.org/10.1016/B978-0-12-398315-2.00006-5

Kleinman, P.J.A., Sharpley, A.N., Withers, P.J.A., Bergström, L., Johnson, L.T. y Doody, D.G., 2015. Implementing agricultural phosphorus science and management to combat eutrophication. En: $A M B I O, 44$, pp.297-310. https://doi.org/10.1007/s13280-015-0631-2 
Kosten, S., Huszar, V.L.M., Bécares, E., Costa, L.S., Van Donk, E., Hansson, L.A., Jeppesen, E., Kruk, C., Lacerot, G., Mazzeo, N., De Meester, L., Moss, B., Lürling, M., Nõges, T., Romo, S. y Scheffer, M., 2012. Warmer climates boost cyanobacterial dominance in shallow lakes. En: Global Change Biology, 18, pp.118-126. https://doi.org/10.1111/j.1365-2486.2011.02488.x

Kozlíková-Zapomělová, E., Ferrari, G. y Del Carmen Pérez, M., 2016. Dolichospermum uruguayense sp. nov., a planktic nostocacean cyanobacterium from the Lower Uruguay River, South America. En: Fottea, 16, pp.189-200. https://doi.org/10.5507/fot.2016.009

Kruk, C., Martínez, A., Martínez De La Escalera, G., Trinchin, R., Manta, G., Segura, Á.M., Piccini, C., Brena, B., Fabiano, G., Pírez, M., Gabito, L., Alcántara, I. y Yannicelli, B., 2019. Exceptional bloom of toxic cyanobacteria on the Uruguayan coast, summer 2019. En: INNOTEC, 18, pp.36-68. https://doi.org/10.26461/18.06

Kruk, C., Mazzeo, N., Lacerot, G. y Reynolds, C., 2002. Classification schemes for phytoplankton: a local validation of a functional approach to the analysis of species temporal replacement. En: Journal of Plankton Research, 24, pp.901-912. https://doi.org/10.1093/plankt/24.9.901

Kruk, C., Segura, A., Nogueira, L., Carballo, C., Martínez De La Escalera, G., Calliari, D., Ferrari, G., Simoens, M., Cea, J., Alcántara, I., Vico, P., Míguez, D. y Piccini, C., 2015. Herramientas para el monitoreo y sistema de alerta de floraciones de cianobacterias nocivas: Río Uruguay y Río de la Plata. En: INNOTEC, 10, pp.23-29.

Kruk, C., Segura, A.M., Nogueira, L., Alcántara, I., Calliari, D., Martínez De La Escalera, G., Carballo, C., Cabrera, C., Sarthou, F., Scavone, P. y Piccini, C., 2017. A multilevel trait-based approach to the ecological performance of Microcystis aeruginosa complex from headwaters to the ocean. En: Harmful Algae, 70, pp.23-36. https://doi.org/10.1016/j.hal.2017.10.004

Lamparelli, M.C., 2004. Grus de trofia en corpos d'água de Estado de Sao Paulo: availacao dos métodos de monitoramento. San Pablo: Universidade de Sao Paulo. (Tesis de Doctorado).

Lazzaro, X., 1997. Do the trophic cascade hypothesis and classical biomanipulation approaches apply to tropical lakes and reservoirs? En: Verhandlungen Internationale Vereinigung für Theoretische und Angewandte Limnologie, 26(2), pp.719-730. https://doi.org/10.1080/03680770.1995.11900811 
Li, J. y Dittrich,M., 2019. Dynamic polyphosphate metabolism in cyanobacteria responding to phosphorus availability. En: Environmental Microbiology, 21, pp.572-583. https://doi.org/10.1111/1462-2920.14488

Li, Y., J. Shang, C. Zhang, W. Zhang, L. Niu, L. Wang, H. Zhang, 2021. The role of freshwater eutrophication in greenhouse gas emissions: A review. En: Science of The Total Environment, 768,

144582, https://doi.org/10.1016/j.scitotenv.2020.144582.

Lizarralde, C., Ciganda, V., Baethgen, W. y Quincke, A., 2016. Pérdida de nutrientes en agua de escurrimiento en sistemas de rotaciones contrastantes. En: Revista INIA, 46, pp.41-43.

http://www.ainfo.inia.uy/digital/bitstream/item/6134/1/Revista-INIAUruguay-n.-46.-p.-41-43.-2016.pdf

Lizarralde, C., Rubio, V. y Sawchik, J., 2014. Relación entre las variables climáticas y del suelo con la pérdida de fósforo por escorrentía [En línea]. En: Congreso Uruguayo de Suelos. Colonia, Uruguay (6-8 de agosto de 2014). Colonia: [s.n.]. [Consulta: 01 de agosto de 2020]. Disponible en: http://www.suelos.com.uy/pdf/74.pdf

Martínez De La Escalera, G., Kruk, C., Segura, A.M., Nogueira, L., Alcántara, I. y Piccini, C., 2017. Dynamics of toxic genotypes of Microcystis aeruginosa complex (MAC) through a wide freshwater to marine environmental gradient. En: Harmful Algae, 62, pp.73-83. https://doi.org/10.1016/j.hal.2016.11.012

Martínez De La Escalera, G., Segura, A.M., Kruk, C., Ghattas, B. y Piccini, C., 2019. Genotyping and functional regression trees reveals environmental preferences of toxic cyanobacteria (Microcystis aeruginosa complex) along a wide spatial gradient. En: bioRxiv. https://doi.org/10.1101/2019.12.20.885111

Mazzeo, N., Clemente, J., García-Rodríguez, F., Gorga, J., Kruk, C., Larrea, D., Meerhoff, M., Quintans, F., Rodríguez-Gallego, L. y Scasso, F., 2002. Eutrofización: causas, consecuencias y manejo. En: Domínguez, A. y Prieto, R.G., eds. Perfil ambiental del Uruguay. Montevideo: Nordan. pp. 39-55. 
Mazzeo, N., Zurbriggen, C., Steffen, M., Barquín, J., Gadino, I., Díaz, I., Ciganda, A. L., Goyenola, G. y Trimble, M., 2019. Descentralización y centralización en la gestión del agua en Uruguay. En: Cardarello, A. y Ferla, P., eds. Descentralización en Uruguay. Propuestas para avanzar en la agenda. Montevideo: Konrad-Adenauer-Stiftung e.V. Fundación Konrad-denauer Oficina Uruguay. pp. 59-72.

Mclellan, N.L. y Manderville, R.A., 2017. Toxic mechanisms of microcystins in mammals. En: Toxicology Research, 6, pp.391-405. https://doi.org/10.1039/c7tx00043j

Meerhoff, M. y Bernardi, R. 2018. Climate Report from Uruguay. En: Performance Research, 23, pp. 56-58. https://doi.org/10.1080/13528165.2018.1495949

Meerhoff, M., Clemente, J.M., Teixeira-De Mello, F., Iglesias, C., Pedersen, A.R. y Jeppesen, E., 2007. Can warm climate-related structure of littoral predator assemblies weaken the clear water state in shallow lakes? En: Global Change Biology, 13, pp.1888-1897. https://doi.org/10.1111/j.13652486.2007.01408.x

Meerhoff, M., Teixeira De Mello, F., Kruk, C., Alonso, C., González-Bergonzoni, I., Pacheco, J. P., Lacerot, G., Arim, M., Beklioglu, M., Brucet, S., Goyenola, G., Iglesias Frizzera, C., Mazzeo, N., Kosten, S. y Jeppensen, E., 2012. 4 Environmental warming in shallow lakes: a review of potential changes in community structure as evidenced from space-for-time substitution approaches. En: Advances in Ecological Research, 46, pp.259-349. https://doi.org/10.1016/B978-0-12-396992-7.00004-6

Modernel, P., Rossing, W.A.H., Corbeels, M., Dogliotti, S., Picasso, V. y Tittonell, P., 2016. Land use change and ecosystem service provision in Pampas and Campos grasslands of southern South America. En: Environmental Research Letters,11, 113002. https://doi.org/10.1088/17489326/11/11/113002

Moss, B., 2008. Water pollution by agriculture. En: Philosophical Transactions of the Royal Society of London B: Biological Sciences, 363, pp.659-666. https://doi.org/10.1098/rstb.2007.2176

Moss, B., Kosten, S., Meerhoff, M., Battarbee, R.W., Jeppesen, E., Mazzeo, N., Havens, K., Lacerot, G., Liu, Z., De Meester, L., Paerl, H. y Scheffer, M., 2011. Allied attack: climate change and nutrient pollution. En: Inland Waters, 1, pp.101-105. https://doi.org/10.5268/IW-1.2.359 
Moss, B., Madgwick, J. y Phillips, G., 1996. A guide to the restoration of nutrient-enriched shallow lakes. Norwich: Broads Authority. 178 pp.

Nagy, G.J., Gómez-Erache, M., López, C.H. y Perdomo, A.C., 2002. Distribution patterns of nutrients and symptoms of eutrophication in the Rio de la Plata River Estuary System. En: Orive, E., Elliott, M. y De Jonge, V.N., eds. Nutrients and eutrophication in estuaries and coastal waters: Proceedings of the 31st Symposium of the Estuarine and Coastal Sciences Association (ECSA), held in Bilbao, Spain, 3-7 July 2000. Dordrecht: Springer. https://doi.org/10.1007/978-94-017-2464-7_10

O'Farrell, I., Motta, C., Forastier, M., Polla, W., Otaño, S., Meichtry, N., Devercelli, M. y Lombardo, R. 2019. Ecological meta-analysis of bloom-forming planktonic Cyanobacteria in Argentina. En: Harmful Algae, 83, pp.1-13. https://doi.org/10.1016/j.hal.2019.01.004

Ockenden, M.C., Hollaway, M.J., Beven, K.J., Collins, A.L., Evans, R., Falloon, P.D., Forber, K. J., Hiscock, K.M., Kahana, R., Macleod, C.J.A., Tych, W., Villamizar, M.L., Wearing, C., Withers, P.J.A., Zhou, J.G., Barker, P.A., Burke, S., Freer, J.E., Johnes, P.J., Snell, M. A., Surridge, B.W.J. y Haygarth, P.M. 2017. Major agricultural changes required to mitigate phosphorus losses under climate change. En: Nature Communications, 8, pp.161. https://doi.org/10.1038/s41467-017-00232-0

Olano, H., Martigani, F., Somma, A. y Aubriot, L., 2019. Wastewater discharge with phytoplankton may favor cyanobacterial development in the main drinking water supply river in Uruguay. En: Environmental Monitoring and Assessment, 191(3), pp.146. https://doi.org/10.1007/s10661-019-7288-4

Olesen, J.E. y Bindi, M., 2002. Consequences of climate change for European agricultural productivity, land use and policy. En: European Journal of Agronomy, 16, pp.239-262. https://doi.org/10.1016/S1161-0301(02)000047

Özen, A., Karapınar, B., Kucuk, İ., Jeppesen, E. y Beklioglu, M., 2010. Droughtinduced changes in nutrient concentrations and retention in two shallow Mediterranean lakes subjected to different degrees of management. En: Hydrobiologia, 646, pp.61-72. https://doi.org/10.1007/s10750-010-0179- x

Paerl,H. y Huisman, J., 2008. Blooms like it hot. En: Science, 320, pp.57-58. https://doi.org/10.1126/science.1155398 
Paerl, H.W., Scott, J.T., Mccarthy, M.J., Newell, S.E., Gardner, W.S., Havens, K.E., Hoffman, D. K., Wilhelm, S.W. y Wurtsbaugh, W.A., 2016. It Takes Two to Tango: When and Where Dual Nutrient (N \& P) Reductions Are Needed to Protect Lakes and Downstream Ecosystems. En: Environmental Science \& Technology, 50, pp.10805-10813.

https://doi.org/10.1021/acs.est.6b02575

Pahl-Wostl, C., 2015. Water governance. Concepts, methods, and practice. Cham: Springer

Penn, C.J., Mcgrath, J.M., Rounds, E., Fox, G. y Heeren, D., 2012. Trapping phosphorus in runoff with a phosphorus removal structure. En: Journal of Environmental Quality, 41, pp.672-679. https://doi.org/10.2134/jeq2011.0045

Perdomo, CH., Barreto, P. y Piñeiro, V., 2015. Pérdida de fósforo desde suelos agrícolas hacia aguas superficiales: resultados preliminares para Uruguay y posibles medidas de manejo para mitigar riesgos. En: Facultad de Agronomía. IV Simposio Nacional de Agricultura. Paysandú, Uruguay (2829 de octubre de 2015). Paysandú: Facultad de Agronomía. pp.77-94.

Peterson, H.M., Baker, L.A., Bruening, D., Nieber, J.L., Ulrich, J.S. y Wilson, B.N., 2017. Agricultural phosphorus balance calculator: a tool for watershed planning. En: Journal of Soil and Water Conservation, 72, pp.395-404. https://doi.org/10.2489/jswc.72.4.395

Piccini, C., Aubriot, L., Fabre, A., Amaral, V., González-Piana, M., Giani, A., Figueredo, C.C., Vidal, L., Kruk, C. y Bonilla, S., 2011. Genetic and ecophysiological differences of South American Cylindrospermopsis raciborskii isolates support the hypothesis of multiple ecotypes. En: Harmful Algae, 10, pp.644-653. https://doi.org/10.1016/j.hal.2011.04.016

Pintos, W., Sommaruga, R., Alfonso, O., Leon, R.D. y Conde, D., 1986. Reporte limnológico: estado de la eutrofización del Lago Jardín (Canelones, Uruguay). Montevideo: Facultad de Ciencias, Secc. Limnología.

Poste, A.E., Hecky, R.E. y Guildford, S.J., 2011. Evaluating microcystin exposure risk through fish consumption. En: Environmental Science \& Technology, 45, pp.5806-5811. https://doi.org/10.1021/es200285c 
Powers, S.M., Bruulsema, T.W., Burt, T.P., Chan, N.I., Elser, J.J., Haygarth, P.M., Howden, N.J.K., Jarvie, H.P., Lyu, Y., Peterson, H.M., Sharpley, Andrew, N., Shen, J., Worrall, F. y Zhang, F., 2016. Long-term accumulation and transport of anthropogenic phosphorus in three river basins. En: Nature Geoscience, 9, pp.353. https://doi.org/10.1038/ngeo2693

Preece, E.P., Hardy, F.J., Moore, B.C. y Bryan, M., 2017. A review of microcystin detections in Estuarine and Marine waters: Environmental implications and human health risk. En: Harmful Algae, 61, pp.31-45. https://doi.org/10.1016/j.hal.2016.11.006

Quinton, J.N., Govers, G., Van Oost, K. y Bardgett, R.D., 2010. The impact of agricultural soil erosion on biogeochemical cycling. En: Nature Geoscience, 3, pp.311-314. https://doi.org/10.1038/ngeo838

Quirós, R., Boveri, M.B., Petracchi, C.A., Rennella, A.M., Rosso, J.J., Sosnovsky, A. y Vonbernard, H.T., 2005. Los efectos de la agriculturización del humedal pampeano sobre la eutrofización de sus lagunas. En: Tundisi, G., Matsumura-Tundisi, T. y Galli, C. S., eds. Eutrofização na América do Sul: Causas, conseqüências e tecnologias de gerenciamento e controle. Rio de Janeiro: Instituto Internacional de Ecologia, Instituto Internacional de Ecologia e Gerenciamento Ambiental, Academia Brasileira de Ciências, Conselho Nacional de Desenvolvimento Científico e Tecnológico. pp.1-16.

Reid, K., Schneider, K. y Joosse, P., 2019. Addressing imbalances in phosphorus accumulation in canadian agricultural soils. En: Journal of Environmental Quality, 48, pp.1156-1166. https://doi.org/10.2134/jeq2019.05.0205

Ruane, E.M., Treacy, M., Mcnamara, K. y Humphreys, J., 2014. Farm-gate phosphorus balances and soil phosphorus concentrations on intensive dairy farms in the south-west of Ireland. En: Irish Journal of Agricultural and Food Research, 53, pp.105-119.

Scasso, F., Mazzeo, N., Gorga, J., Kruk, C., Lacerot, G., Clemente, J., Fabian, D. y Bonilla, S., 2001. Limnological changes in a sub-tropical shallow hypertrophic lake during its restoration: two years of a whole-lake experiment. En: Aquatic Conservation: Marine and Freshwater Ecosystems, 11, pp.31-44. https://doi.org/10.1002/aqc.420 
Schärer, M., Stamm, C., Vollmer, T., Frossard, E., Oberson, A., Flühler, H. y Sinaj, S., 2007. Reducing phosphorus losses from over-fertilized grassland soils proves difficult in the short term. En: Soil Use and Management, 23, pp154-164. https://doi.org/10.1111/j.1475-2743.2007.00114.x

Schindler, D., 2006. Recent advances in the understanding and management of eutrophication. En: Limnology and Oceanography, 51, pp.356-363. https://doi.org/10.4319/lo.2006.51.1_part_2.0356

Schindler, D.W., Carpenter, S.R., Chapra, S.C., Hecky, R.E. y Orihel, D.M. 2016. Reducing phosphorus to curb Lake Eutrophication is a success. En: Environmental Science \& Technology, 50, pp.8923-8929. https://doi.org/10.1021/acs.est.6b02204

Sharpley, A.N., Bergström, L., Aronsson, H., Bechmann, M., Bolster, C.H., Börling, K., Djodjic, F., Jarvie, H.P., Schoumans, O.F., Stamm, C., Tonderski, K.S., Ulén, B., Uusitalo, R. y Withers, P.J.A., 2015. Future agriculture with minimized phosphorus losses to waters: Research needs and direction. En: Ambio, 44, pp.163-179. https://doi.org/10.1007/s13280-014-0612-x

Sharpley, A.N., Mcdowell, R.W. y Kleinman, P.J.A., 2001. Phosphorus loss from land to water: integrating agricultural and environmental management. En: Plant and Soil, 237, pp.287-307. https://doi.org/10.1023/A:1013335814593

Sims, J.T., Simard, R.R. y Joern, B.C., 1998. Phosphorus loss in agricultural drainage: historical perspective and current research. En: Journal of Environmental Quality, 27, pp.277-293. https://doi.org/10.2134/jeq1998.00472425002700020006x

Smith, D.R., Huang, C. y Haney, R.L., 2017. Phosphorus fertilization, soil stratification, and potential water quality impacts. En: Journal of Soil and Water Conservation, 72, pp.417-424. https://doi.org/10.2489/jswc.72.5.417

Smith, D.R., King, K.W. y Williams, M.R., 2015. What is causing the harmful algal blooms in Lake Erie? En: Journal of Soil and Water Conservation, 70, pp.27A-29A. https://doi.org/10.2489/jswc.70.2.27A

Smith, V.H., Joye, S.B. y Howarth, R.W., 2006. Eutrophication of freshwater and marine ecosystems. En: Limnology and Oceanography, 51, pp.351-355. https://doi.org/10.4319/lo.2006.51.1_part_2.0351 
Sommaruga, R., Conde, D. y Casal, J.A., 1995. The role of fertilizers and detergents for eutrophication in Uruguay. En: Fresenius Environmental Bulletin, 4, pp.111-116.

Søndergaard, M., Jensen, J.P. y Jeppesen, E., 2003. Role of sediment and internal loading of phosphorus in shallow lakes. En: Hydrobiologia, 506, pp.135145. https://doi.org/10.1023/B:HYDR.0000008611.12704.dd

Steffen, M. e Inda, H., eds., 2010. Bases técnicas para el manejo integrado de Laguna del Sauce y cuenca asociada. Montevideo: Universidad de la República, South American Institute for Resilience and Sustainability Studies. 150 pp.

Stutter, M.I., Chardon, W.J. y Kronvang, B., 2012. Riparian buffer strips as a multifunctional management tool in agricultural landscapes: introduction. En: Journal of Environmental Quality, 41, pp.297-303. https://doi.org/10.2134/jeq2011.0439

Taranu, Z.E., Gregory-Eaves, I., Leavitt, P.R., Bunting, L., Buchaca, T., Catalan, J., Domaizon, I., Guilizzoni, P., Lami, A., Mcgowan, S., Moorhouse, H., Morabito, G., Pick, F.R., Stevenson, M.A., Thompson, P.L. y Vinebrooke, R.D., 2015. Acceleration of cyanobacterial dominance in north temperatesubarctic lakes during the Anthropocene. En: Ecology Letters, 18, pp.375384. https://doi.org/10.1111/ele.12420

Teixeira De Mello, F., Meerhoff, M., Pekcan-Hekim, Z. y Jeppesen, E., 2009. Substantial differences in littoral fish community structure and dynamics in subtropical and temperate shallow lakes. En: Freshwater Biology, 54, pp.1202-1215. https://doi.org/10.1111/j.1365-2427.2009.02167.x

Testai, E., Scardala, S., Vichi, S., Buratti, F.M. y Funari, E., 2016. Risk to human health associated with the environmental occurrence of cyanobacterial neurotoxic alkaloids anatoxins and saxitoxins. En: Critical Reviews in Toxicology, 46, pp.385-419.

https://doi.org/10.3109/10408444.2015.1137865

Trimble, M., Jacobi, P., Olivier, T., Pascual, M., Zurbriggen, C., Garrido, L. y Mazzeo, N., 2020. Reconfiguring water governance for resilient socialecological systems in South America. En: Baird, J. y Plummer, R., eds. Water resilience. Cham: Springer Nature. pp. 113-135. 
Trimble, M. y Mazzeo, N., 2020. Water governance for adaptation and resilience in the Anthropocene. En: Torres, P., Jacobi, P.R., BarbiI, J. y Goncalves, L., eds. Adaptation and public policies at the Sao Paulo macrometropolis: a science-policy approach. São Paulo: Letra Capital Editora. pp. 29-33.

Unión Europea. Directiva 2000/60/CE del Parlamento Europeo y el Consejo de 23 de octubre de 2000 por la que se establece un marco comunitario de actuación en el ámbito de la política de aguas. Diario Oficial de la Comunidad Europea, 22 de diciembre de 2000, L 327, pp.72.

Uruguay. Decreto 253/979, de 9 de mayo de 1979. Diario Oficial, 31 de mayo de 1979, p.1479.

Uruguay. Decreto 284/990, de 21 de junio de 1990. Diario Oficial, 25 de julio de 1990, p.649.

Uruguay. Decreto 333/004, de 16 de setiembre de 2004. Diario Oficial, 22 de setiembre de 2004, p.671.

Uruguay. Decreto 405/008, de 21 de agosto de 2008. Diario Oficial, 27 de agosto de 2008, p.645.

Uruguay. Ley 15.239, de 23 de diciembre de 1981. Diario Oficial, 07 de enero de 1982, p.1976.

Uruguay. Ley 19.553, de 27 de octubre de 2017. Diario Oficial, 16 de noviembre de 2017 , p. 5.

Uruguay. Ministerio de Ganadería Agricultura y pesca, DIEA, 2019. Anuario estadístico agropecuario. Montevideo: MGAP.

Uruguay. Ministerio de Vivienda Ordenamiento Territorial y Medio Ambiente, DINAMA, 2020. Indicadores ambientales: agua, concentración de fósforo total [En línea]. Montevideo: DINAMA. [Consulta: 30 de abril de 2020]. Disponible en:

https://www.dinama.gub.uy/indicadores_ambientales/ficha/oanconcentracion-de-fosforo-total/

Vidal, F., Sedan, D., D'agostino, D., Cavalieri, M.L., Mullen, E., Parot Varela, M.M., Flores, C., Caixach, J. y Andrinolo, D., 2017. Recreational exposure during algal bloom in carrasco beach, Uruguay: A Liver Failure Case Report. En: Toxins, 9, pp.267. https://doi.org/10.3390/toxins9090267 
Vidal, L. y Britos, A., 2012. Uruguay: occurrence, toxicity and regulation of Cyanobacteria [En línea]. En: Chorus, I., ed. Current approaches to Cyanotoxin risk assessment, risk management and regulations in different countries. Dessau: Federal Environmental Agency (Umweltbundesamt). [Consulta:30 de abril de 2020]. Disponible en: https://www.umweltbundesamt.de/publikationen/current-approaches-tocyanotoxin-risk-assessment-0

Vitousek, P.M., Cassman, K., Cleveland, C., Crews, T., Field, C.B., Grimm, N.B., Howarth, R. W., Marino, R., Martinelli, L., Rastetter, E.B. y Sprent, J.I., 2002. Towards an ecological understanding of biological nitrogen fixation. En: Boyer, E.W. y Howarth, R.W., eds. The nitrogen cycle at regional to global scales. Dordrecht: Springer Netherlands.

Whitton, B.A., 2012. Ecology of cyanobacteria II: their diversity in space and time. Berlín: Springer. https://doi.org/10.1007/978-94-007-3855-3 